\title{
Resultados del tratamiento del nervio radial e influencia de factores pronósticos
}

\section{The Outcomes of Radial Nerve Injury Treatment and Influence of Prognostic Factors}

\author{
Gregorio Martínez Villén ${ }^{1}$ Sami Hamam Alcober ${ }^{1} \quad$ Rafael Cardona Malfey $^{1}$ \\ ${ }^{1}$ Departamento de Cirugía Ortopédica y Traumatología, Unidad de \\ Cirugía Reconstructiva Del Aparato Locomotor, Mano-microcirugía, \\ Hospital Universitario Miguel Servet, Zaragoza, España \\ Rev Iberam Cir Mano 2017;45:104-114. \\ Address for correspondence Gregorio Martínez Villén, MD, PhD, \\ Departamento de Cirugía Ortopédica y Traumatología, Unidad de \\ Cirugía Reconstructiva Del Aparato Locomotor, Mano-microcirugía, \\ Hospital Universitario Miguel Servet, 5009 Zaragoza, España \\ (e-mail: gmartinezvillen@gmail.com).
}

\section{Resumen}

Palabras Clave

- lesiones nervio radial

- tratamiento

- pronóstico
Objetivo Analizar la respuesta de las lesiones del nervio radial y de sus ramas al tratamiento y la influencia de factores asociados dependientes del tipo de daño y de paciente.

Material y Método Sesenta y seis pacientes con lesiones del nervio radial o de sus ramas terminales fueron sometidos a tratamiento conservador, reconstrucción nerviosa, exoneurólis, recolocación nerviosa, cobertura con colgajo graso o transferencia tenomuscular. Hubo 39 hombres y 27 mujeres con una edad media de 45 años. Valoramos la situación motora antes y después del tratamiento con la escala del Louisiana State University Health Sciences Centre (G0 a G5). En las lesiones de la rama superficial del nervio radial fueron recogidas las alteraciones sensitivas y el dolor. La edad, el momento de la cirugía, el tipo de lesión, su nivel y tratamiento, el tiempo de seguimiento y el momento aproximado de la recuperación nerviosa, fueron analizados y relacionados con los resultados.

Resultados Hubo 37 lesiones del nervio radial entre la axila y el codo, 12 del nervio interóseo posterior y 17 de la rama sensitiva superficial. En 10 pacientes observamos recuperación espontánea. En el 82,3\% de los casos de reconstrucción del nervio radial el resultado fue bueno o excelente, siendo desfavorable en el $17,7 \%$. Todos los pacientes con reconstrucción del nervio interóseo posterior tuvieron resultado excelente. Las lesiones nerviosas en continuidad tuvieron un $20 \%$ más de resultados G 4 y G 5 que las neurotmesis. Entre los pacientes con lesión de la rama sensitiva superficial, seis quedaron asintomáticos y 11 sintomáticos después del tratamiento. Los pacientes de mayor edad, los grandes defectos nerviosos injertados en las zonas más proximas de la extremidad superior, o con mayor tiempo transcurrido entre la lesión y la reparación evolucionaron peor.

Conclusión El nervio radial es un nervio agradecido al tratamiento.

Background The purpose of this study was to present the response of radial nerve lesions and their branches to treatment, observing the influence of associated factors depending to the damage and the patient. received

July 10,2017

accepted

September 18, 2017

published online

November 3, 2017
DOI https://doi.org/

10.1055/s-0037-1607459.

ISSN 1698-8396.
Copyright $\odot 2017$ Thieme Revinter

Publicações Ltda, Rio de Janeiro, Brazil

License terms

c) $(1) \$$ 
Methods Sixty-six patients with injuries of the radial nerve or its terminal branches had conservative treatment, nerve reconstruction, exoneurolysis, nerve relocation, fat flap or tendon transfer. Thirty-nine were men and 27 women, with a mean age of 45 years. Motor status of the patients was estimated before and after treatment using the Louisiana State University Health Sciences Center scale (G0 to G5). Sensory disturbances or residual pain were recorded for superficial sensory radial nerve injuries. Patient age, timing of surgery, type of lesion and treatment, level of injury, follow-up, and the approximative time of onset of motor recovery were estimated and related to the results.

Results There were 37 injuries of the radial nerve between the axilla and elbow, 12 injuries of the posterior interosseous nerve and 17 injuries of the radial sensory nerve. Spontaneous recovery was observed in ten patients. There were $82.3 \%$ favorable and $17.7 \%$ poor outcomes after radial nerve reconstruction. All patients with posterior interosseus nerve reconstruction had good or excellent outcomes. Patients with incontinuity injuries had $20 \%$ more G4 and G5 outcomes than those with neurotmesis. In superficial sensory radial nerve injuries, six patients were asymptomatic and 11

\section{Keywords}

- radial nerve injuries

- treatment

- prognostic symptomatic after treatment. Older patients, large nerve defects treated with grafts in the proximal areas of the upper limb, or longer time between the injury and repair, evolved worse.

Conclusion The radial nerve is grateful to treatment.

\section{Introducción}

En la práctica clínica, las lesiones del nervio radial(NR) o de sus ramas interósea posterior (NIOP) y sensitiva radial superficial (NSRS), se encuentran entre las más comunes de la extremidad superior. El mayor porcentaje de casos con parálisis radial tiene su origen en fracturas del tercio medio o distal del húmero., ${ }^{1,2}$ La parálisis del NIOP en el antebrazo es menos frecuente. Hasta el 35\% de las lesiones del NR y del NIOP son iatrogénicas, ${ }^{3-6}$ particularmente después de la reducción abierta y fijación de fracturas de húmero y radio. ${ }^{5,7}$ La compresión tumoral del nervio como causa de daño es rara, usualmente producida por lipomas o gangliones. ${ }^{8-10}$ El déficit sensitivo en el territorio subsidiario del NSRS puede aparecer por lesión de esta rama terminal o del tronco común del NR, asociando dolor neuropático discapacitante. ${ }^{11,12}$

Como en la mayoría de los nervios periféricos, el pronóstico de las lesiones del NR y de sus ramas terminales varía según la edad, el tipo y el nivel de la lesión, el periodo transcurrido entre la misma y su reparación, o la técnica quirúrgica empleada. ${ }^{13-15}$ La correlación de todos estos factores es difícil, por lo que su influencia en los resultados del tratamiento ha sido analizada en pocas ocasiones. ${ }^{16}$ Sin embargo, el carácter exclusivamente motor (o casi) del NR y del NIOP, y su recorrido en la extremidad superior relativamente corto hasta la placa motora de los músculos que inervan, conceden a sus lesiones un pronóstico en teoría esperanzador. ${ }^{2,5,9,16-19}$ No ocurre lo mismo con las lesiones del NSRS, cuya evolución es menos predecible. ${ }^{11,12,18}$

El propósito de ese trabajo es estudiar la interacción de factores que pueden modificar el pronóstico y los resultados del tratamiento conservador o quirúrgico tras distintos tipos de lesión del NR o de sus ramas. Se intentará en último término dar respuesta a la siguiente pregunta: ¿es el NR realmente agradecido al tratamiento?

\section{Material y Método}

\section{Población de Estudio}

Sesenta y seis pacientes con lesiones del NR o de sus ramas fueron tratados consecutivamente por el primer autor (GMV), en el mismo centro hospitalario entre Junio de 2003 y Agosto de 2016. Treinta y nueve fueron hombres y 27 mujeres, con edades comprendidas entre los cuatro meses y los 82 años (media de 45). El NR estuvo comprometido en 37 pacientes, el NIOP en 12 y el NSRS en 17. En 41 ocasiones se vio afectado el lado derecho y en 25 el izquierdo. El origen de la lesión se presenta en la - Tabla 1.

La obtención de los datos se efectuó revisando las historias clínicas y valorando la situación en las exploraciones previas y posteriores al tratamiento, hasta que el paciente fue dado de alta con un resultado considerado definitivo. Los pacientes fueron revisados una media de 6,6 (3-11) veces en el posoperatorio, estimando de manera aproximada el inicio de la recuperación motora. Todos los afectados fueron informados sobre su patología y las alternativas de tratamiento seleccionadas, dando la conformidad para la ejecución de los procedimientos terapéuticos y su seguimiento.

\section{Criterios de Inclusión}

1. Hombres y mujeres independientemente de la edad, con lesión diagnosticada del NRo de sus ramas, simple o asociada a otros nervios o estructuras anatómicas adyacentes. 
Tabla 1 Causas de la lesión y número de afectados

\begin{tabular}{|l|l|l|l|}
\hline \multicolumn{2}{|l|}{ Nervio lesionado ( ${ }^{\circ}$ lesiones) } \\
\hline & NR & NIOP & NSRS \\
\hline Fractura de húmero & 18 & & \\
\hline Fractura de radio & & 3 & \\
\hline Laceración & 7 & & 3 \\
\hline latrogénica & 8 & 7 & 9 \\
\hline Contusión & 2 & & \\
\hline Herida de bala & 1 & & \\
\hline Compresión tumoral & 1 & 2 & 2 \\
\hline Atrapamiento cicatriz & & & 3 \\
\hline Total & 37 & 12 & 17 \\
\hline
\end{tabular}

Abreviaciones: NR, nervio radial; NIOP, nervio interóseo posterior; NSRS, nervio sensitivo radial superficial.

2. Pacientes sometidos a tratamiento conservador o quirúrgico.

3. Lesiones de origen traumático, iatrogénico o por compromiso tumoral.

\section{Criterios de Exclusión}

1. Neuropatías degenerativas, inflamatorias inespecíficas o idiopáticas.

2. Síndromes canaliculares primarios tipo arcada de Fröshe, salvo lesión iatrogénica derivada de una intervención quirúrgica previa para la descompresión del nervio.

\section{Evaluación Clínica}

Los estudios de conducción nerviosa fueron realizados antes y después del tratamiento en los casos con afectación del NR o del NIOP; o durante la intervención quirúrgica cuando se observó una lesión en continuidad. En las lesiones quirúrgicas atendidas en urgencia, los electromiogramas solo se hicieron en el postoperatorio. Los registros eléctricos y la exploración física pre o intraoperatoria, nos permitieron situar el nivel topográfico de la lesión según los criterios Pan y cols. ${ }^{17}$ Para el NR: nivel I infraclavicular, nivel II en el surco espiral del húmero, nivel III entre el surco espiral y la articulación del codo. Para el NIOP: nivel IV en el antebrazo. La exploración física de la función motora pre y posoperatoria fue valorada con la graduación del Louisiana State University Health Sciences Centre (-Tabla 2). ${ }^{18}$

En la literatura consultada, no hemos encontrado escalas validadas para la tipificación de los hallazgos sensitivos pre y posoperatorios en las lesiones del NSRS. Por ese motivo, y dada la escasa utilidad que tiene la estimación de la sensibilidad discriminativa a dos puntos en el dorso de la mano, decidimos registrar la existencia de anestesia, hipoestesia o disestesia.

\section{Elección del Tratamiento}

El tratamiento conservador se decidió cuando el mecanismo de acción del daño y las pruebas diagnósticas del preoperatorio sugerían lesiones en continuidad con un potencial de recuperación espontánea posible. Si esa recuperación no tuvo lugar transcurrido un periodo medio de cuatro meses, que se entendió "seguro" para una hipotética actuación quirúrgica sobre el nervio, practicamos cirugía de revisión.

Los procedimientos quirúrgicos realizados fueron: reconstrucción nerviosa mediante sutura termino-terminal, termino-lateral o injerto nervioso; exoneurólisis; resección de neuroma y recolocación nerviosa; cobertura con colgajo graso y transferencia tenomuscular. Algunos procedimientos como la exoneurólisis se asociaron a otros en un mismo tiempo quirúrgico. La elección de la técnica quirúrgica para el NR y el NIOP se hizo según el algoritmo descrito en la - Fig. 1.

Realizamos los injertos nerviosos siguiendo el procedimiento habitual cuando no se pudo conseguir una

Tabla 2 Graduación de la función motora según el Louisiana State University Health Sciences Centre ${ }^{18}$ para lesiones del nervio radial y del nervio interóseo posterior

\begin{tabular}{|l|l|l|l|}
\hline Grado & Evaluación & Lesión NR & Lesión NIOP \\
\hline 0 & ausente & Sin función NR & Sin función NIOP \\
\hline 1 & pobre & $\begin{array}{l}\text { Indicios de contracción triceps. Ausencia o indicios de } \\
\text { contracción BR solo contra gravedad. }\end{array}$ & $\begin{array}{l}\text { Indicio de contracción ECU } \\
\text { solo contra gravedad; } \\
\text { ausencia EDC y EPL }\end{array}$ \\
\hline 2 & justa & $\begin{array}{l}\text { Contracción triceps y BR contra resistencia. Ausencia o } \\
\text { indicio de supinación. No extensión de muñeca. }\end{array}$ & $\begin{array}{l}\text { Recuperación ECU, ausencia } \\
\text { o indicio en EDC o EPL, } \\
\text { o ambos }\end{array}$ \\
\hline 3 & moderada & $\begin{array}{l}\text { Contracción triceps y BR contra resistencia. Supinación y } \\
\text { extensión de muñeca contra gravedad. Ausencia o } \\
\text { indicio de EPL y EDC. }\end{array}$ & $\begin{array}{l}\text { Recuperación ECU y algo de } \\
\text { EDC. Ausencia o indicio de } \\
\text { EPL }\end{array}$ \\
\hline 5 & buena & $\begin{array}{l}\text { Contracción triceps, BR, supinación y extensión muñeca } \\
\text { contra resistencia. Indicio o mayor EDC y EPL }\end{array}$ & $\begin{array}{l}\text { Recuperación total ECU, y } \\
\text { moderada del EDC y EPL }\end{array}$ \\
\hline 5 & excelente & $\begin{array}{l}\text { Buen triceps, BR, supinación y extensión de muñeca. EPL } \\
\text { y EDC al menos contra gravedad y alguna Resistencia. }\end{array}$ & $\begin{array}{l}\text { Recuperación total de ECU, } \\
\text { EDC y EPL }\end{array}$ \\
\hline
\end{tabular}

Abreviaciones: BR, brachioradialis; ECU, extensor carpi ulnaris; EDC, extensor digitorum communis; EPL, extensor pollicis longus; NIOP, nervio interóseo posterior; NR, nervio radial.

Tabla adaptada en su traducción y abreviaturas, tomada de Kim DH, Kam AC, Chandika P, Tiel RL, Kline DG. Surgical management and outcome in patients with radial nerve lesions. J Neurosurg 2001; 95: 573-83. 


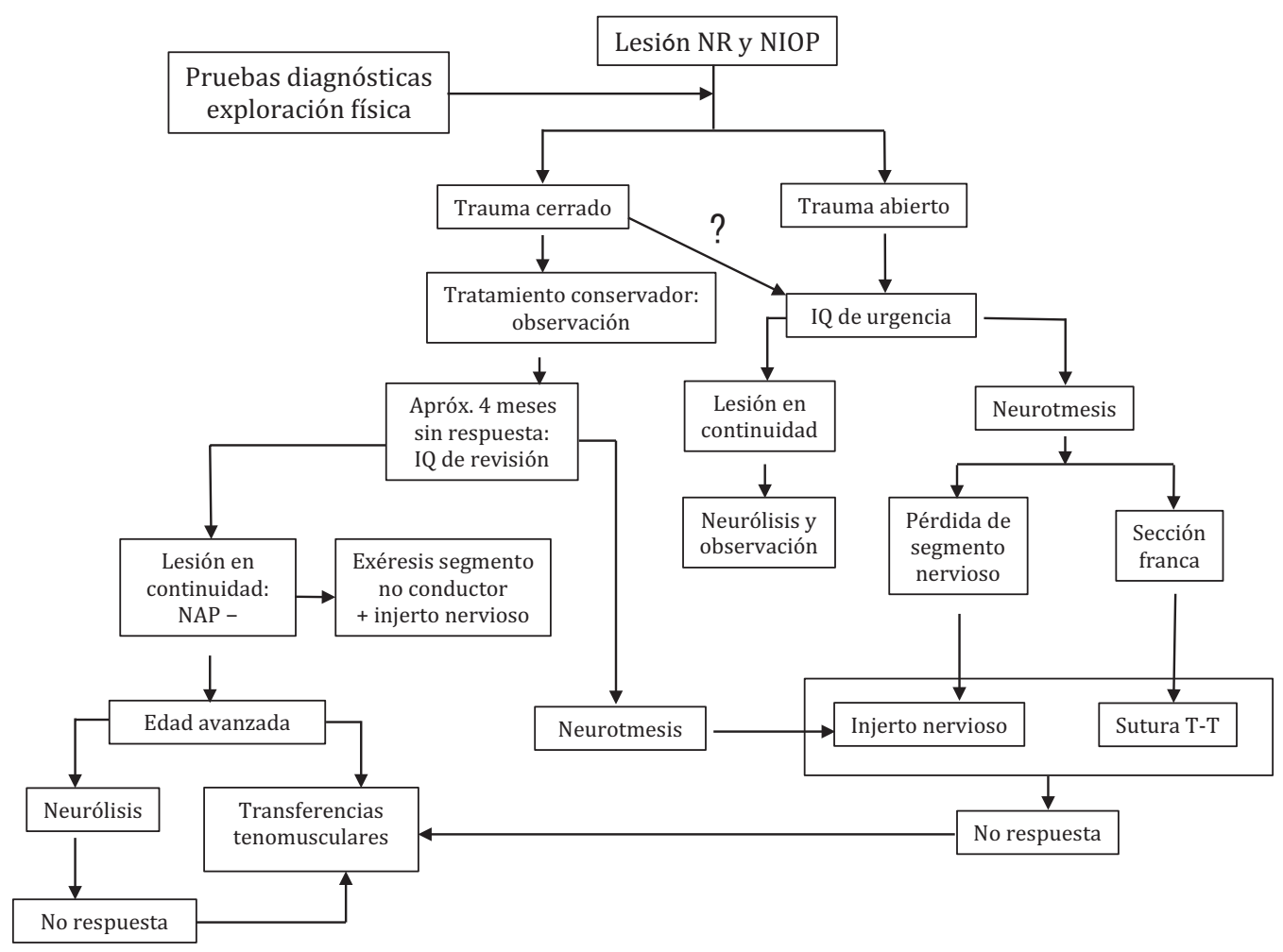

Fig. 1 Algoritmo para el tratamiento de las lesiones del nervio radial y del nervio interóseo posterior. Abreviaciones: IQ, intervención quirúrgica; NIOP, nervio interóseo posterior; NR, nervio radial; T-T, termino-terminal.

sutura directa sin tensión. El nervio sural se empleó para los injertos fasciculados del NR y del NIOP, mientras que el nervio cutáneo medial del antebrazo fue destinado a los injertos simples del NSRS. La coaptación de los cabos de sección se realizó con puntos sueltos de sutura de $9 / 0$ ó 10/0, protegiendo finalmente la neurorráfia con Tissucol ${ }^{\circledR}$ (Baxter, Viena, Austria). Todas las intervenciones que exigieron reconstrucción nerviosa y/o neurólisis se hicieron con medios de magnificación óptica, procurando dejar el nervio reparado en un lecho bien vascularizado. El tiempo medio transcurrido entre la lesión y el momento de la intervención quirúrgica fue de 3,6 (0-7) meses en los casos de reconstrucción nerviosa, y de 4,4 (0-8) meses en los de neurólisis.

Las transferencias tenomusculares se realizaron por imposibilidad o fracaso de la reconstrucción nerviosa o del tratamiento conservador; por estar sobrepasado el tiempo recomendable para la reconstrucción nerviosa, o en pacientes de edad avanzada. Los resultados de las transferencias tenomusculares no son objeto de este trabajo.

\section{Resultados}

Treinta y nueve nervios se hallaron totalmente seccionados (neurotmesis), y 27 tuvieron una lesión en continuidad, tres de ellos con neuromas intermedios. Esa situación condujo a la intervención quirúrgica en 56 pacientes, cuyas características se describen en la - Tabla 3. En el grupo de reconstrucción nerviosa hubo tres reparaciones primarias, cuatro primarias diferidas y 17 secundarias. Diez pacientes recibieron tratamiento conservador.

\section{Nervios Radial e Interóseo Posterior}

Antes del tratamiento observamos dos casos G0 en nivel I (parálisis del NR completa); 12 casos G0 en nivel IV (parálisis del NIOP completa), y 31 casos GI en niveles II y III (solo se conservaba la extensión del codo).

En líneas generales, en las 36 lesiones del NR y del NIOP sometidas a reconstrucción, neurólisis o tratamiento

Tabla 3 Tipo de tratamiento y número de procesos

\begin{tabular}{|l|l|l|l|}
\hline \multirow{2}{*}{} & \multicolumn{3}{|l|}{$\begin{array}{l}\text { Nervio lesionado } \\
\left(\mathbf{n}^{\circ} \text { de procesos }\right)\end{array}$} \\
\cline { 2 - 4 } & NR & NIOP & NSRS \\
\hline Anastomosis T-T & 2 & 0 & 5 \\
\hline Anastomosis T-L & 0 & 0 & 1 \\
\hline Injerto nervioso (cm) & 10 & 4 & 2 \\
\hline Exoneurólisis & 9 & 1 & 5 \\
\hline Resección-recolocación & 0 & 0 & 3 \\
\hline Colgajo graso & 0 & 0 & 1 \\
\hline $\begin{array}{l}\text { Tratamiento } \\
\text { conservador }\end{array}$ & 7 & 3 & 0 \\
\hline $\begin{array}{l}\text { Transferencia } \\
\text { tendinosa }\end{array}$ & 9 & 4 & 0 \\
\hline Total & 37 & 12 & 17 \\
\hline
\end{tabular}

Abreviaciones: NIOP, nervio interóseo posterior; NR, nervio radial; NSRS, nervio sensitivo radial superficial; T-L, anastomosis termino-lateral; T-T, anastomosis termino- terminal. 

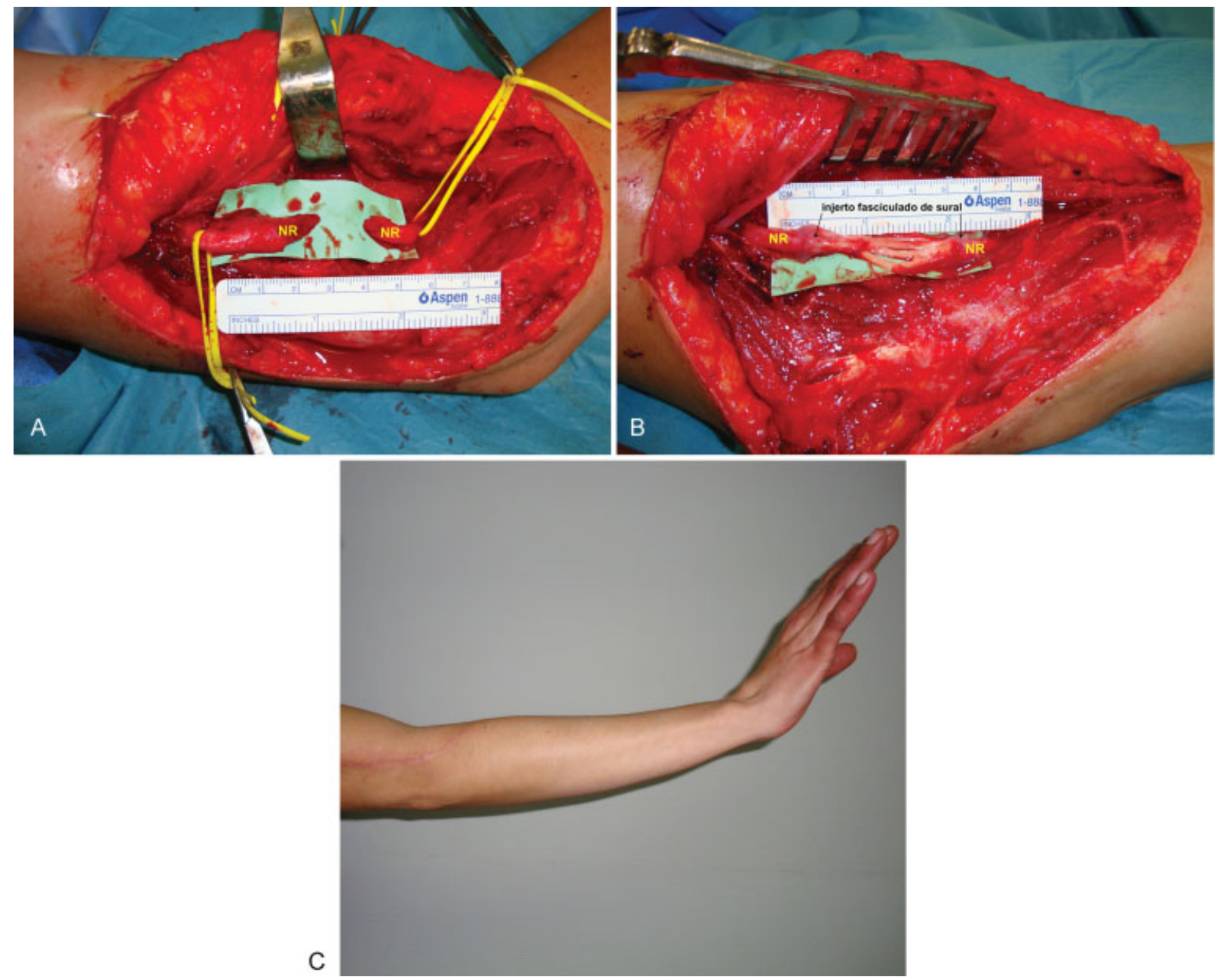

Fig. 2 Paciente de 28 años de edad. Parálisis radial persistente 6 meses después de la osteosíntesis de una fractura diafisometafisara distal de húmero. (A) Neurotmesis del radial encontrada en la cirugía de revisión (NR). (B) Tratamiento con un injerto fasciculado de $4 \mathrm{~cm}$. (C) Imagen a los 12 meses de la reconstrucción mostrando recuperación completa de la función.

conservador obtuvimos los siguientes resultados: 22 casos con recuperación grado $\mathrm{V}$ (61\%); 13 de ellos con lesiones en continuidad y neurólisis, o recuperación espontánea, y nueve después de la reconstrucción por sección (-Figs. 2 y 3). Nueve casos alcanzaron un grado IV (25\%), 6 de ellos con lesiones en continuidad y neurólisis o recuperación espontánea, y tres después de la reconstrucción por sección. Dos casos alcanzaron un grado III (5,5,\%), ambos después de la reconstrucción por sección. Otros dos casos $(5,5 \%)$ tuvieron un grado II, uno de ellos tras recuperación espontánea, y otro después de la reconstrucción por sección. Finalmente un paciente tuvo un grado $0(2,7 \%)$ tras la reconstrucción por sección. De las lesiones en continuidad del NR, tres fueron exploradas quirúrgicamente obteniendo un potencial de acción nervioso (PAN) negativo entre los niveles II y III, siendo sometidas a neurólisis: dos de ellas se

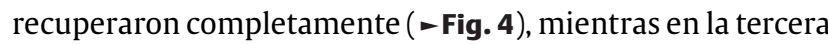
hubo una función de grado II.

El seguimiento medio posterior al tratamiento con reconstrucción del NR o del NIOP fue de 24,6 (15-28) meses; de 16,8 (7-32) meses en pacientes solo con neurólisis, y de 9,2 (5-15) meses en pacientes con recuperación espontánea. La relación entre la edad, momento de la cirugía, tipo de tratamiento, nivel de la lesión y tiempo de seguimiento se muestran en las - Tablas $\mathbf{4}$ y $\mathbf{5}$.

El inicio de la recuperación motora en el NR con injertos de una longitud media de 4,2 (3-5) cm, fue de 7,6 (6-9) meses. En esa valoración no se ha tenido en cuenta un injerto de $8 \mathrm{~cm}$ que resultó fallido. Para el NIOP con una longitud media de los injertos de 3,5 (3-4) cm, fue de 6,2 (6-7) meses. Con la neurorráfia termino-terminal del NR la recuperación comenzó de media a los 5,5 (5-6) meses. La recuperación del NR después de la neurólisis o de manera espontánea aconteció de media a los 4,4 (3-7) meses, y antes de los 3 meses para el NIOP.

\section{Nervio Sensitivo Radial Superficial}

Dos de las lesiones del NSRS fueron halladas en el antebrazo proximal y 15 en el antebrazo distal-muñeca. En la - Tabla 1 se pueden ver las causas de la lesión, destacando por sus características la compresión por un glomangioma $(1 \times 1 \mathrm{~cm})$ y un liposarcoma $(7 \times 4,4 \times 3,5 \mathrm{~cm})$ (-Fig. 5). Las lesiones iatrogénicas procedían de tenólisis por tenosinovitis de De Quervain, de exéresis de gangliones o de osteosíntesis por fractura de radio. 

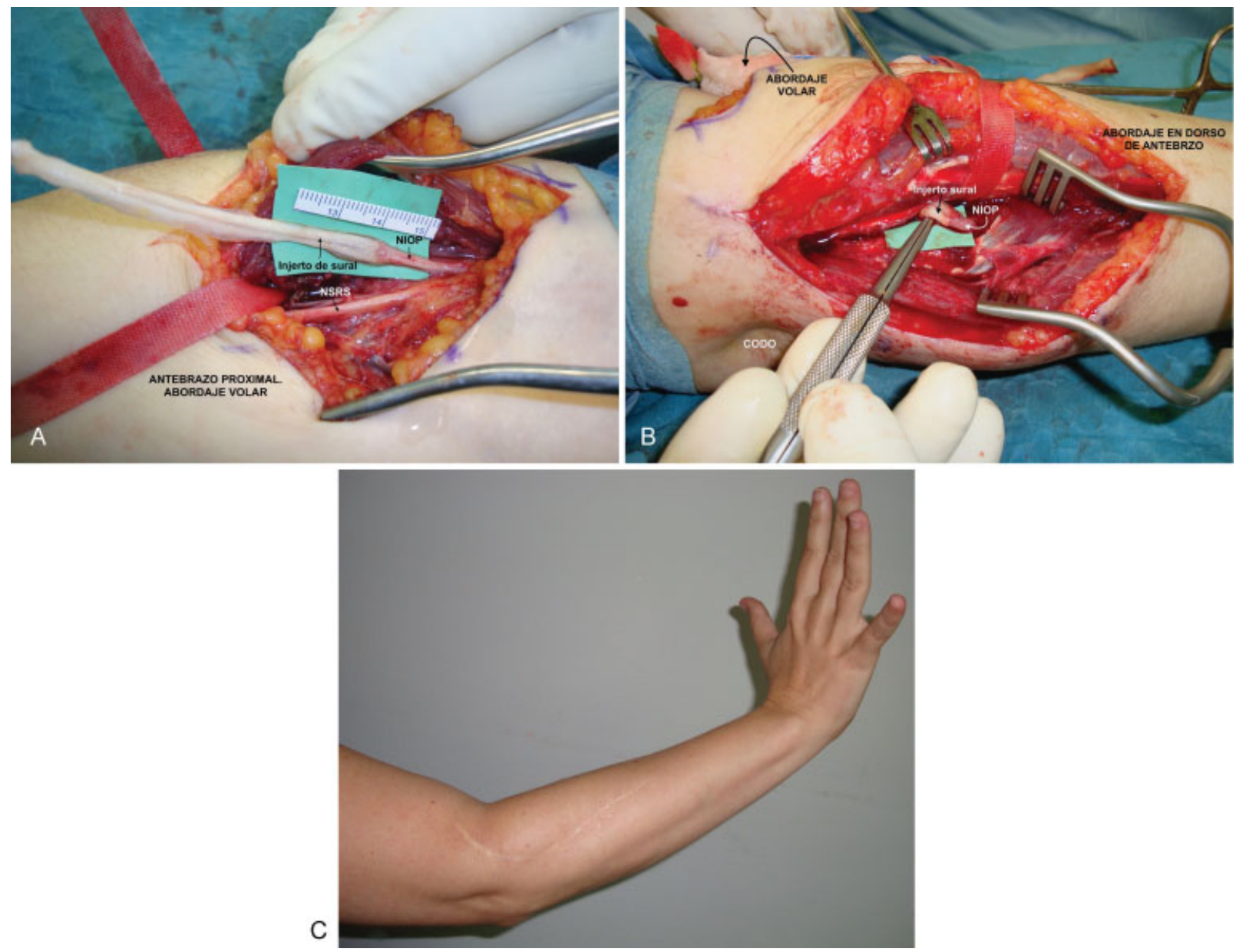

Fig. 3 Paciente de 39 años de edad. Parálisis del interóseo posterior persistente 5,5 meses después de la osteosíntesis de una fractura de cabeza de radio. Neurotmesis del interóseo posterior encontrada en la cirugía de revisión y tratada con un injerto de sural por doble vía de abordaje, volar (A) y dorsal (B). (C) Imagen a los 20 meses de la reconstrucción mostrando recuperación completa de la función. Abreviaciones: NIOP, nervio interóseo posterior; NSRS, nervio sensitivo radial superficial.

Antes del tratamiento todos los pacientes presentaban zonas de anestesia e hipoestesia irregularmente distribuidas en la vertiente dorsal y radial de la mano; ocho de ellos con dolor neuropático intenso próximo a una cicatriz quirúrgica. Uno de estos pacientes había sido intervenido en diez ocasiones con resecciones sucesivas del NSRS (- Fig. 6). Las características generales de la técnica quirúrgica destinada a las lesiones del NSRS se encuentran descritas en la -Tabla 3. Uno de los casos con neurólisis fue también sometido a un colgajo graso de cobertura ( - Fig. 7 ).

El seguimiento medio posterior al tratamiento con reconstrucción del NSRS fue 19,3 (3-24) meses. Al finalizar dicho seguimiento, seis pacientes estaban asintomáticos; dos después de la anastomosis termino-terminal, y cuatro después de la neurólisis, incluidos los dos casos de extirparción tumoral. Once pacientes permanecieron sintomáticos: seis con alteraciones sensitivas y dolor residual, y cinco con parestesias; uno de ellos con hiperestesia ante estímulos mínimos.

\section{Discusión}

Las lesiones del NR o de sus ramas representan un 23,9\% de todas las lesiones de las ramas terminales del plexo braquial operadas por nosotros en un periodo de 13 años. La etiología y el tratamiento en nuestra casuística no han sido muy distintos de lo que hay publicado en la literatura consultada. En cuanto a la influencia de los factores pronósticos en el tratamiento, hemos encontrado coincidencias y discrepancias con otros autores, que expondremos a continuación. A pesar de que nuestro trabajo presenta aspectos metodológicos mejorables, pensamos que los resultados pueden ser útiles para el enfoque terapéutico de esos pacientes.

\section{Neurotmesis en el Radial e Interóseo Posterior}

Después de la reconstrucción del NR alcanzamos buenos o excelentes resultados en el $82,3 \%$ de los casos, y pobres o malos en el $17,7 \%$. Con el mismo tipo de lesión, todas las reconstrucciones del NIOP tuvieron excelentes resultados. Estos hallazgos son similares a otros ya publicados. ${ }^{9,16,17}$

En el momento actual se acepta ampliamente una relación inversamente proporcional entre la edad y la capacidad de recuperación de un nervio. ${ }^{6,20}$ Dicha relación se encuentra estrechamente vinculada a factores como el potencial de regeneración y la plasticidad cerebral, muy desarrollada en los niños pero limitada en el adulto. ${ }^{21}$ En cualquier caso, determinar qué pacientes han de ser operados con técnicas 


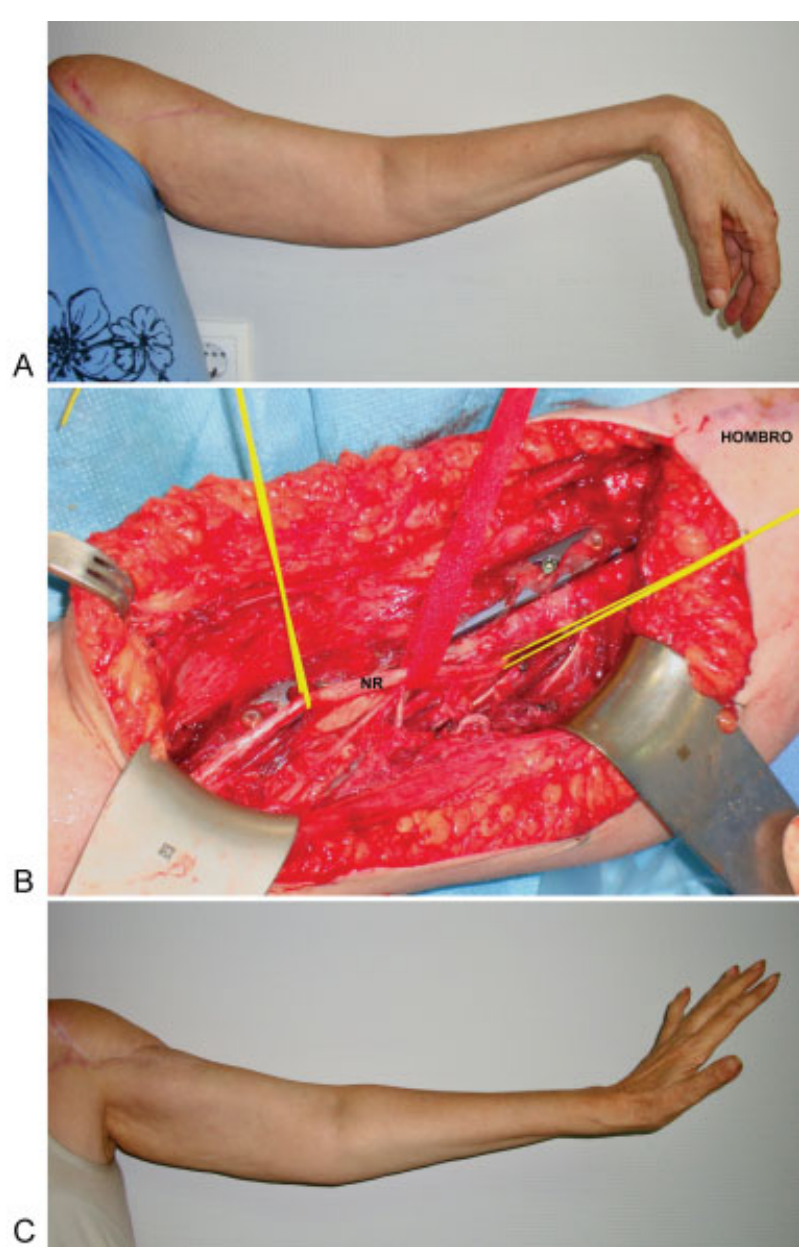

Fig. 4 Paciente de 57 años de edad. (A). Típica mano y dedos caídos tras una parálisis radial después de la ostesíntesis en urgencia de una fractura de húmero proximal. (B) Cirugía de revisión 5 meses después de la oseosíntesis mostrando el NR en continuidad, pero potencial de acción nervioso negativo. Se practicó extensa neurólisis. (C) Imagen a los 7 meses de la neurólis mostrando recuperación completa de la función. Abreviaciones: NR, nervio radial.

de reconstrucción atendiendo exclusivamente a la edad, constituye una decisión controvertida. Incluso en personas de edad avanzada, el dolor neuropático causado por un neuroma de sección puede aliviarse o desaparecer gracias a la reordenación que experimentan los axones al reconstruir la continuidad del nervio.

En nuestro trabajo, los extremos de edad del grupo con neurotmesis del radial o del interóseo posterior fueron de 22 y 59 años. En los pacientes con recuperación G5 (máxima posible), la media de edad fue 36,2 años: 32,5 para las lesiones del NR, y 43,6 para las del NIOP. Los resultados G3 y G4 se consiguieron en pacientes con edad media de 25,8 años; es decir, más jóvenes que los del grupo G5. Esto pudo deberse a que los últimos presentaron lesiones neurovasculares asociadas. Kallio y cols, ${ }^{19}$ y Pan y cols. ${ }^{17}$ observaron que los resultados del tratamiento no estaban relacionados con la edad de los pacientes de sus respectivos estudios, con valores medios en torno a los 29,5 años.

En nuestra serie, el tipo de reconstrucción nerviosa estuvo fuertemente asociado al resultado funcional. En los casos con
Tabla 4 Resultados según la graduación del Louisiana State University Health Sciences Centre (LSUHS) ${ }^{18}$ en 16 pacientes con neurotmesis del radial (NR) y del interóseo posterior (NIOP), y su relación con los valores medios de edad, momento de la cirugía, longitud del injerto nervioso, nivel de la lesión según Pan y col. ${ }^{17}$ y tiempo de seguimiento.

\begin{tabular}{|l|l|l|l|l|l|l|}
\hline \multirow{2}{*}{} & \multicolumn{2}{|l|}{ LSUHS } \\
\cline { 2 - 8 } & G0 & G1 & G2 & G3 & G4 & G5 \\
\hline Casos & 1 & 0 & 1 & 2 & 3 & 9 \\
\hline Edad (años) & 42 & & 59 & 27,5 & 24,6 & 36,2 \\
\hline $\begin{array}{l}\text { Momento de la } \\
\text { cirugía (meses) }\end{array}$ & 6 & & 0,5 & 2 & 5,3 & 2,3 \\
\hline $\begin{array}{l}\text { Injerto nervioso } \\
\text { (cm) }\end{array}$ & 8 & & 3,5 & 4,5 & 3 & 3 \\
\hline Nivel I (NR) & & & 1 & & & \\
\hline Nivel II (NR) & & & & & 2 & \\
\hline Nivel III (NR) & 1 & & & 2 & & 6 \\
\hline Nivel IV (NIOP) & & & & & 1 & 3 \\
\hline $\begin{array}{l}\text { Seguimiento } \\
\text { (meses) }\end{array}$ & 13 & & 47 & 21,5 & 12 & 27,7 \\
\hline
\end{tabular}

Tabla 5 Resultados según la graduación del Louisiana State University Health Sciences Centre (LSUHS) ${ }^{18}$ en 20 pacientes con lesiones en continuidad del nervio radial (NR) y del nervio interóseo posterior (NIOP), y su relación con los valores medios de edad, tipo de tratamiento, momento de la cirugía, longitud del injerto nervioso, nivel de la lesión según Pan y cols. ${ }^{17}$ y tiempo de seguimiento.

\begin{tabular}{|l|l|l|l|l|l|l|}
\hline \multirow{2}{*}{} & \multicolumn{3}{|l}{ LSUHS } & \multicolumn{5}{l|}{} \\
\cline { 2 - 7 } & G0 & G1 & G2 & G3 & G4 & G5 \\
\hline Casos & 0 & 0 & 1 & 0 & 6 & 13 \\
\hline Edad (años) & & & 64 & & 55 & 50,3 \\
\hline $\begin{array}{l}\text { Recuperación } \\
\text { espontánea }\end{array}$ & & & & & 4 & 7 \\
\hline Exoneurólisis & & & 1 & & 1 & 7 \\
\hline $\begin{array}{l}\text { Momento de } \\
\text { la cirugía }\end{array}$ & & & 0 & & 8 & 3,2 \\
\hline Nivel I (NR) & & & & & & 1 \\
\hline Nivel II (NR) & & & 1 & & 6 & 4 \\
\hline Nivel III (NR) & & & & & & 4 \\
\hline Nivel IV (NIOP) & & & & & & 4 \\
\hline $\begin{array}{l}\text { Seguimiento } \\
\text { (meses) }\end{array}$ & & & 16 & & 14,7 & 13,5 \\
\hline
\end{tabular}

laceración neta del NR y anastomosis termino-terminal hecha en urgencia, la recuperación fue más rápida y completa. Solo obtuvimos resultados similares en pacientes cuya reconstrucción se hizo con injertos de menos de $3 \mathrm{~cm}$ de longitud. El único caso con resultado G0 tenía 42 años y fue operado con un injerto de $8 \mathrm{~cm}$ seis meses 


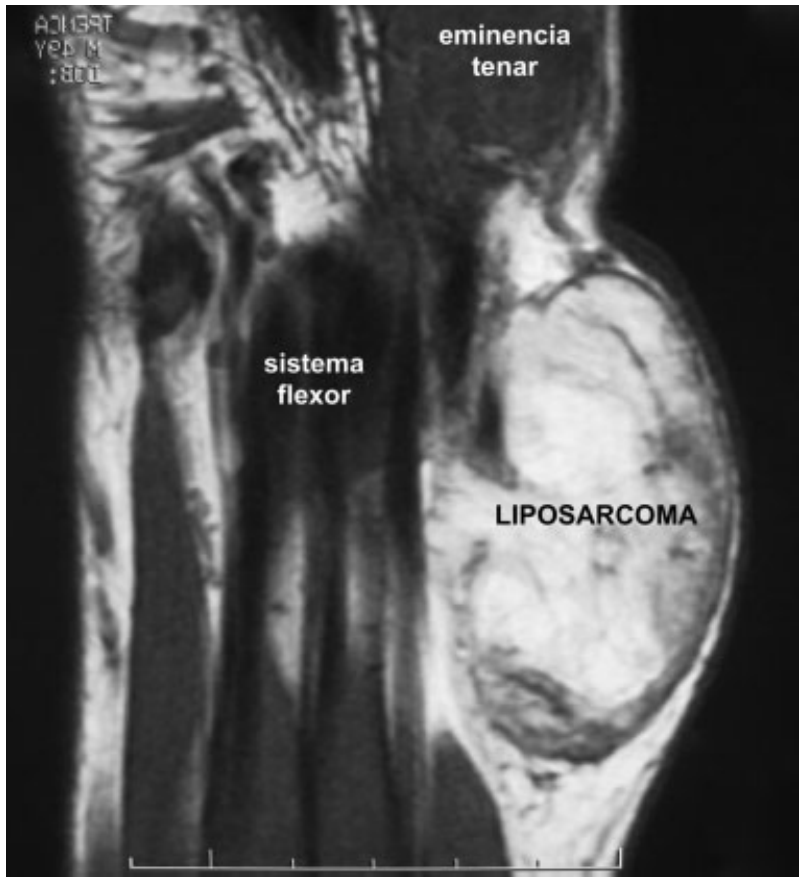

Fig. 5 Paciente de 48 años de edad. Imagen de resonancia magnética mostrando un liposarcoma sobre el trayecto del nervio sensitivo radial superficial, al que comprimía.

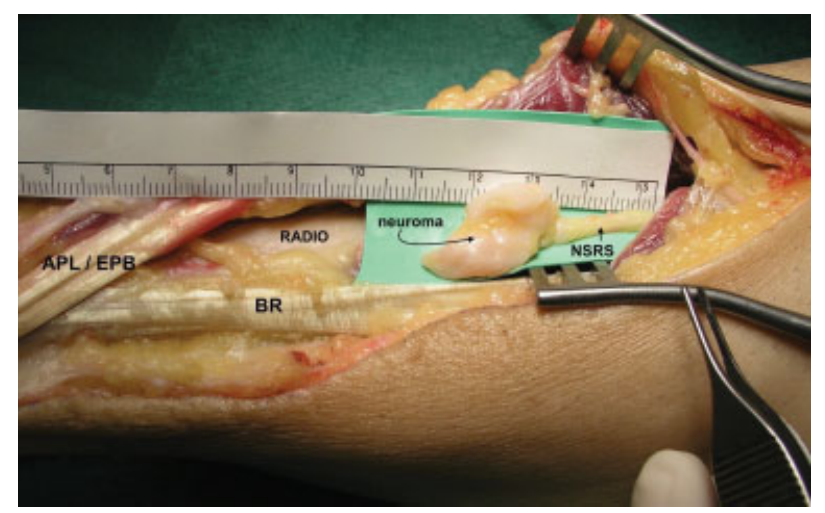

Fig. 6 Paciente de 55 años de edad. Neuroma del sensitivo radial superficial extirpado en 10 intervenciones distintas. Abreviaciones: APL, abductor pollicis longus; BR, brachioradialis; EPB, extensor pollicis brevis; NSRS, nervio sensitivo radial superficial.

después de una fractura de húmero englobando el nervio. La discrepancia en el número de pacientes de nuestro estudio con sutura termino-terminal o injerto, fue debida a que la mayor parte se intervino varias semanas después de la lesión. Eso implica retracción en los cabos de sección, lo que hace inviable la sutura termino-terminal sin tensión.

Para la mayoría de los autores, ${ }^{16,19,22,23}$ los mejores resultados, cuando hubo pérdida de tejido nervioso, se lograron con injertos de hasta $5 \mathrm{~cm}$, ya que la orientación de las fibras regeneradas es más fácil y rápida. ${ }^{24}$ En un estudio reciente de Bertelli y Ghizoni utilizando injertos con una media de $12,5(8-18) \mathrm{cm}$ de longitud en lesiones muy proximales del NR, los autores tuvieron resultados que no eran "ideales". ${ }^{25}$ Pan y cols. ${ }^{17}$ lograron buenos resultados con injertos nerviosos de longitud media de $6,4 \mathrm{~cm}$,
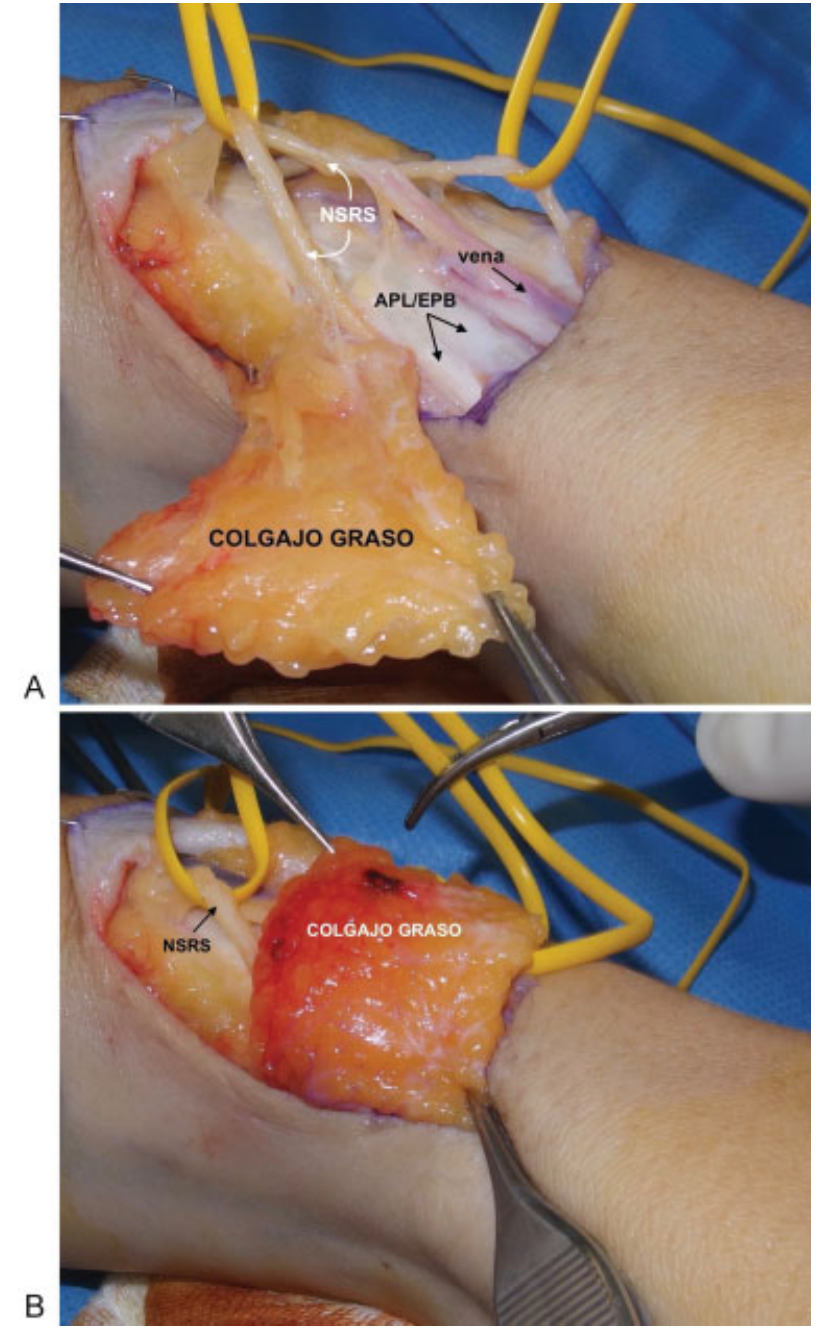

Fig. 7 Paciente de 31 años de edad. Hiperestesia después de tres intervenciones quirúrgicas fallidas por una tenosinovitis de De Quervain. (A) Neurólisis del sensitivo radial superficial, y (B) disección de un colgajo graso para cubrir el nervio. Abreviaciones: APL, abductor pollicis longus; EPB, extensor pollicis brevis; NSRS, nervio sensitivo radial superficial.

excluyendo de la reconstrucción aquellos casos con pérdida nerviosa mayor de $15 \mathrm{~cm}$. Habitualmente el nervio donante de elección para injertos fasciculados es el sural. Con menor calibre, el nervio cutáneo antebraquial medial puede ser tomado para la reconstrucción del NSRS.

En lo que concierne al nivel de la lesión, los peores resultados funcionales los observamos cuando el daño del NR tuvo lugar en las zonas más proximales (niveles I y II de Pan y cols. ${ }^{17}$ ). En Estos casos, la fibra nerviosa regenerada ha de recorrer una distancia muy larga entre el punto de la lesión y la placa motora de destino, que puede ser refractaria a la reinervación cuando llegan los axones. Este fenómeno usualmente coincide con la atrofia muscular, que aparece entre 15 y 18 meses después del daño. Por otro lado, cuanto más proximal es la lesión, mayor es la dispersión de fibras y la cantidad de tejido conjuntivo nervioso. Estos hechos pueden explicar por qué ante una lesión similar, el NIOP responde generalmente mejor que el NR.

En nuestros pacientes, la reconstrucción precoz del NR o del NIOP fue más favorable funcionalmente, encontrando 
resultados G5 en casos con una media de 2,3 meses transcurridos entre la lesión y la reparación quirúrgica. La reconstrucción temprana evita o reduce la fibrosis y la retracción de los cabos de sección, y permite una identificación mejor de los grupos fasciculares. Además, el potencial metabólico del nervio es óptimo entre 15 y 21 días después de la lesión, ${ }^{15}$ y la placa motora es más probable que responda a la reinervación.

En nuestro estudio, el nivel de la lesión y el momento de la cirugía han mantenido una fuerte relación, de ahí la respuesta al tratamiento en casos como el siguiente: mujer diabética de 59 años con recuperación del triceps M4 después de un injerto en el NR de 3,5 cm en nivel I, tras 15 días del accidente. El resto de la función nerviosa de la extremidad fue nula a pesar de la reparación de los nervios mediano y cubital y de la arteria humeral, que también estaban seccionados. ${ }^{26}$

Establecer el momento de la recuperación motora no es fácil. En las lesiones del NR reparadas en nivel II tiene lugar a los 5 o 6 meses, necesitando entre 3 y 4 meses las que ocurren en un nivel III. 7,13,27 Para Kim y cols., en un estudio de 45 pacientes con lesiones del NIOP de los cuales 15 eran neurotmesis, el momento de la recuperación tuvo lugar entre 5 y 7 meses. ${ }^{9}$ Nosotros comprobamos el inicio de la respuesta a los 7,6 meses de media para el NR injertado, y a los 6,2 meses para el NIOP.Un paciente de 22 años con una laceración iatrogénica del NR en zona II, recuperó la extensión de la muñeca 9 meses después de un injerto nervioso de $3,5 \mathrm{~cm}$, alcanzando el máximo grado de función en el conjunto de la mano 18 meses después de la cirugía. La respuesta electromiográfica de la recuperación en ese tipo de pacientes, normalmente precede a la que proporciona la exploración física al menos en un mes. ${ }^{6,9,18,28}$ En nuestros pacientes, la validez del EMG para esa valoración fue poco clara o incluso contradictoria.

\section{Lesiones en Continuidad de los Nervios Radial e Interóseo Posterior}

Las lesiones en continuidad de los nervios son las que mejor pronóstico tienen. ${ }^{16,18,28}$ En la parálisis del NR por neuroapraxia asociada a fracturas del húmero, el 70,7\% de los casos se recuperarán espontáneamente. ${ }^{2}$ La función completa de la extremidad puede alcanzarse entre 3 y 5,4 meses, según se haya practicado cirugía de revisión o tratamiento conservador. ${ }^{1}$ Nosotros vimos un 20\% más de recuperación grado 4 y 5 en lesiones en continuidad para el grupo de pacientes de 50 años.

La elección del tratamiento es controvertida cuando el PAN intraoperatorio de una lesión en continuidad es negativo. Algunos autores resecan el segmento cuya conducción eléctrica es nula y lo reconstruyen. ${ }^{18}$ En nuestra opinión, eso es muy arriesgado si se hace poco tiempo después del accidente; o improcedente en casos con edad avanza, en los que preferimos primero la neurólisis $\mathrm{y}$, si fracasa, las transferencias tenomusculares. De nuestros tres pacientes con lesiones del NR en continuidad y PAN intraoperatorio negativo, dos se recuperaron liberando el nervio, mientras el tercero tuvo un resultado G2. Se trataba de un hombre de 64 años con neurotmesis radial por arma de fuego en nivel II, asociada a la sección de la arteria humeral y del nervio mediano; contusión del nervio cubital y fractura conminuta del húmero. La cirugía paliativa con transferencias tenomusculares no fue posible en este caso por parálisis definitiva mediano-cubital y rigideces articulares.

\section{Lesiones del Nervio Sensitivo Radial Superficial}

La trascendencia funcional del NSRS en la mano es escasa, pero sus lesiones no son inocuas. El trayecto anatómico de este nervio hace que sea vulnerable a traumatismos y percances iatrogénicos. Dos estudios diferentes de Atherton y cols., y Birch en un total de 471 neuromas dolorosos de los nervios cutáneos de la extremidad superior, revelaron que el NSRS estuvo implicado en el 58,8\% y el 32,3\% respectivamente. Un $53,8 \%$ de las intervenciones destinadas a su reparación fue producto de lesiones iatrogénicas. ${ }^{11,13}$ Las consecuencias fueron neuritis, atrapamiento en una cicatriz y neuromas de sección 0 en continuidad, con el resultado de severas alteraciones sensitivas y dolor neuropático de muy difícil tratamiento., 3,11,12 Casi la mitad de las lesiones del NSRS en nuestra casuística procedían de la cirugía por tenosinovitis de De Quervain, de las cuales el 30\% habían sido operadas más de dos veces. Una causa menos común de lesión del NSRS fue la compresión nerviosa por tumores grasos, gangliones o glomangiomas, cuya escisión conduce habitualmente a mejores resultados que cuando se procede a la reconstrucción o neurólisis cicatricial. ${ }^{10}$ En contraste con los buenos resultados de la cirugía en el NR y el NIOP, la recuperación de las lesiones del NSRS es menos favorable. ${ }^{5}$ Kin y cols. comunican un $76 \%$ de casos con mejoría significativa del dolor neuropático después de la reconstrucción quirúrgica del nervio, sin hacer referencia a la recuperación sensitiva. ${ }^{18}$ Calfee y cols. encontraron que en un 56\% de sus casos operados mediante neurolisis no hubo disestesia, aunque un $44 \%$ permanecieron sintomáticos. ${ }^{12}$ Terzis y Konofaos lograron buenos o excelentes resultados sensitivos en el 76,9\% de sus pacientes sometidos a la reconstrucción, con una mejoría excelente del dolor en el $69,2 \%$, y buena en el $23 \%{ }^{16}$ En nuestra serie, el 35,3\% de los pacientes quedaron asintomáticos después de la cirugía, mientras el $64,7 \%$ presentaron algún síntoma. En ese sentido conviene resaltar que nosotros valoramos como resultado desfavorable tanto la persistencia de alteraciones sensitivas como del dolor. A pesar de ello, observamos que los afectados se manifestaron satisfechos cuando el dolor se alivió, concediendo escasa importancia a las alteraciones sensitivas residuales. En el paciente al que se había resecado un neuroma del sensitivo radial en 10 ocasiones previas, lo localizamos en el tercio medio del antebrazo con un tamaño de $1 \mathrm{~cm}$. Después de extirparlo, el extremo resultante se dividió en dos segmentos que fueron anastomosados entre sí, enterrando el nervio en el brachiorradialis según la técnica propuesta por Atherton y cols. $^{11}$ El territorio subsidiario del NSRS permaneció insensible, pero el severo dolor neuropático que el paciente soportaba desapareció.

\section{Resultados del Tratamiento del Nervio Radial Frente a Otros Nervios de la Extremidad Superior}

La mayoría de los estudios que han comparado los resultados funcionales del tratamiento de los nervios mediano, cubital y radial han demostrado que la respuesta del NR es mejor. ${ }^{29-32}$ 
Sin embargo, estos trabajos no tuvieron en cuenta la influencia de posibles sesgos causados por suplencias funcionales mediano-cubital (anastomosis de MartinGruber); o la posibilidad de que el paciente valorado tuviese una mano completamente inervada por el cubital. ${ }^{33}$

Aunque en este trabajo no ha sido motivo de estudio la repuesta comparativa entre los tres nervios, nuestra experiencia refleja que con similares características de lesión nerviosa y de tratamiento, el NR siempre mostró una evolución más favorable que la del mediano, y mucho más que la del cubital. Ese resultado obedece al siguiente argumento: el NR es un nervio esencialmente motor, lo que reduce el riesgo de una reinervación cruzada sensitivomotora tras la anastomosis, siendo aprovechado mucho más el contingente axonal en crecimiento. Por otro lado, los músculos subsidiarios del NR tienen una función sinérgica muy selectiva, que requiere una coordinación menos exigente que la de los nervios mediano y cubital; eso hace que también se reduzca el riesgo de reinervación entre músculos antagonistas. Finalmente la distancia menor entre el lugar de la lesión y la placa motora de destino, favorece una regeneración más rápida que la de los nervios mediano y cubital. ${ }^{7,32}$

\section{¿Transferencias Tenomusculares o Reconstrucción Nerviosa?}

Según Ingary y Green, las transferencias tenomusculares en la parálisis del NR o del NIOP "se encuentran entre las mejores y más predecibles de la extremidad superior". ${ }^{27}$ Esa filosofía condujo en su momento a algunos autores a considerar las transferencias tendinosas de elección; incluso ante casos en los que la reconstrucción se podía realizar en condiciones favorables para ofrecer resultados satisfactorios. $^{34}$ Las transferencias tenomusculares constituyen una opción excelente si la reparación nerviosa fracasa, es imposible o está contraindicada.

La recuperación funcional efectiva de la mano en la parálisis del NR después de transferencias tenomusculares es una media de 6 a 8 meses más rápida que la de una reconstrucción nerviosa exitosa, aunque esta última proporciona mejores resultados biomecánicos y de coordinación motora. ${ }^{16,17,23,27,35}$ A pesar de todo, algunos estudios muestran que el déficit funcional después de las transferencias tenomusculares en la parálisis radial es muy pequeño para las actividades de la vida diaria. ${ }^{36}$

En una lesión del NR en zona I sin complicaciones asociadas, la recuperación de la extensión del codo es predecible después de la reparación nerviosa, pero muy dudosa la recuperación de la extensión de la muñeca, de las metacarpofalángicas y del pulgar, ${ }^{17,25}$ como sucedió en uno de nuestros pacientes. En esos casos, autores como Bertelli y Ghizoni rechazan los injertos nerviosos como única alternativa, proponiendo neurotizaciones o transferencias tenomusculares asociadas. ${ }^{25}$ En el momento actual no hay consenso en la elección entre una neurotización o una transferencia tendinosa; o la realización de ambas simultáneamente. Los argumentos dependen no solo de la obtención de una adecuada respuesta funcional; también del riesgo de condicionar una transferencia tenomuscular si fracasa la neurotización. Otra ventaja de las transferencias tenomusculares en la parálisis del NR o del NIOP viene dada por la posibilidad de llevarlas a cabo sin un límite de tiempo siempre que la calidad del tendón y del músculo donantes sean adecuadas y no haya rigideces articulares en la mano. Brown describió una transferencia tenomuscular satisfactoria después de 24 años de una parálisis radial. ${ }^{37}$ Nosotros logramos muy buena movilidad de la mano en dos pacientes con parálisis del NR y del NIOP 52 y 30 años después de la lesión original. ${ }^{38}$

\section{Conclusiones}

Nuestros resultados comparados con los encontrados en la literatura consultada, ponen de relieve que las lesiones del NR y del NIOP responden mayoritariamente bien a la reconstrucción quirúrgica o al tratamiento conservador. Las características anatomofuncionales de estos nervios pueden contribuir a ello, aunque la presencia de determinados factores pronósticos condicionará los resultados de cualquier tratamiento. A pesar de todo, incluso en pacientes con fracaso del tratamiento primario, la cirugía paliativa mediante transferencias tenomusulares, suele proporcionar muy buenos resultados. Esto nos permite concluir que el NR es un nervio agradecido al tratamiento. Desafortunadamente, las lesiones del NSRS son menos predecibles.

\section{Conflicto de Interes}

Los autores declaran que no hay conflictos de intereses.

\section{Bibliografía}

1 Korompilias AV, Lykissas MG, Kostas-Agnantis IP, Vekris MD, Soucacos PN, Beris AE. Approach to radial nerve palsy caused by humerus shaft fracture: is primary exploration necessary? Injury 2013;44(03):323-326

2 Shao YC, Harwood P, Grotz MR, Limb D, Giannoudis PV. Radial nerve palsy associated with fractures of the shaft of the humerus: a systematic review. J Bone Joint Surg Br 2005;87(12):1647-1652

3 Khan R, Birch R. Latropathic injuries of peripheral nerves. J Bone Joint Surg Br 2001;83(08):1145-1148

4 Kretschmer T, Antoniadis G, Braun V, Rath SA, Richter HP. Evaluation of iatrogenic lesions in 722 surgically treated cases of peripheral nerve trauma. J Neurosurg 2001;94(06):905-912

5 Kretschmer T, Heinen CW, Antoniadis G, Richter HP, König RW. latrogenic nerve injuries. Neurosurg Clin N Am 2009;20(01):73-90, vii

6 Wang JP, Shen WJ, Chen WM, Huang CK, Shen YS, Chen TH. Iatrogenic radial nerve palsy after operative management of humeral shaft fractures. J Trauma 2009;66(03):800-803

7 Adams JE, Steinmann SP. Nerve injuries about the elbow. J Hand Surg Am 2006;31(02):303-313

8 Fitzgerald A, Anderson W, Hooper G. Posterior interosseous nerve palsy due to parosteal lipoma. J Hand Surg [Br] 2002;27(06): 535-537

9 Kim DH, Murovic JA, Kim YY, Kline DG. Surgical treatment and outcomes in 45 cases of posterior interosseous nerve entrapments and injuries. J Neurosurg 2006;104(05):766-777

10 Martínez-Villén G, Badiola J, Alvarez-Alegret R, Mayayo E. Nerve compression syndromes of the hand and forearm associated with tumours of non-neural origin and tumour-like lesions. J Plast Reconstr Aesthet Surg 2014;67(06):828-836

11 Atherton DD, Fabre J, Anand P, Elliot D. Relocation of painful neuromas in Zone III of the hand and forearm. J Hand Surg Eur Vol 2008;33(02):155-162 
12 Calfee RP, Shin SS, Weiss AP. Neurolysis of the distal superficial radial nerve for dysaesthesia due to nerve tethering. J Hand Surg Eur Vol 2008;33(02):152-154

13 Birch R. Nerve repair. En: Wolfe SW, Hotchkins RN, Pederson WC, Kozin SH (Ed). Green's operative hand surgery, 6th ed. Philadelphia: Elservier Churchill Livingstone; 2011:1035-1074

14 Daneyemez M, Solmaz I, Izci Y. Prognostic factors for the surgical management of peripheral nerve lesions. Tohoku J Exp Med 2005; 205(03):269-275

15 Lundborg G. A 25-year perspective of peripheral nerve surgery: evolving neuroscientific concepts and clinical significance. J Hand Surg Am 2000a25(03):391-414

16 Terzis JK, Konofaos P. Radial nerve injuries and outcomes: our experience. Plast Reconstr Surg 2011;127(02):739-751

17 Pan $\mathrm{CH}$, Chuang DC, Rodríguez-Lorenzo A. Outcomes of nerve reconstruction for radial nerve injuries based on the level of injury in 244 operative cases. J Hand Surg Eur Vol 2010;35(05):385-391

$18 \mathrm{Kim}$ DH, Kam AC, Chandika P, Tiel RL, Kline DG. Surgical management and outcome in patients with radial nerve lesions. J Neurosurg 2001;95(04):573-583

19 Kallio PK, Vastamäki M, Solonen KA. The results of secondary microsurgical repair of radial nerve in 33 patients. J Hand Surg [Br] 1993;18(03):320-322

20 Birch R, Achan P. Peripheral nerve repairs and their results in children. Hand Clin 2000;16(04):579-595

21 Lundborg G. Brain plasticity and hand surgery: an overview. J Hand Surg [Br] 2000b25(03):242-252

22 Matejcík V. Peripheral nerve reconstruction by autograft. Injury 2002;33(07):627-631

23 Shergill G, Bonney G, Munshi P, Birch R. The radial and posterior interosseous nerves. Results fo 260 repairs. J Bone Joint Surg $\mathrm{Br}$ 2001;83(05):646-649

24 Millesi H. Nerve grafting. En: Slutsky DJ, Hentz VR (Ed). Peripheral nerve surgery: practical applications in the upper extremity. Philadelphia: Elservier Churchill Livingstone; 2006:39-59

25 Bertelli JA, Ghizoni MF. Results of nerve grafting in radial nerve injuries occurring proximal to the humerus, including those within the posterior cord. J Neurosurg 2015;124:1-7

26 Martínez Villén G. Caso clínico. En Llusa M, Palazzi S, Valer A(Ed). Anatomía quirúrgica del plexo braquial y de los nervios periféricos de la extremidad superior. Madrid: Panamericana SA; 2013:113

27 Ingary JV, Green DP. Radial nerve palsy. En: Wolfe SW, Hotchkins RN, Pederson WC, Kozin SH (Ed). Green's operative hand surgery, 6th ed. Philadelphia: Elservier Churchill Livingstone; 2011: 1075-1092

28 Postacchini F, Morace GB. Fractures of the humerus associated with paralysis of the radial nerve. Ital J Orthop Traumatol 1988;14 (04):455-464

29 Kim DH, Han K, Tiel RL, Murovic JA, Kline DG. Surgical outcomes of 654 ulnar nerve lesions. J Neurosurg 2003;98(05):993-1004

30 Taha A, Taha J. Results of suture of the radial, median, and ulnar nerves after missile injury below the axilla. J Trauma 1998;45 (02):335-339

31 Roganovic Z, Pavlicevic G. Difference in recovery potential of peripheral nerves after graft repairs. Neurosurgery 2006;59 (03):621-633, discussion 621-633

32 Murovic JA. Upper-extremity peripheral nerve injuries: a Louisiana State University Health Sciences Center literature review with comparison of the operative outcomes of 1837 Louisiana State University Health Sciences Center median, radial, and ulnar nerve lesions. Neurosurgery 2009;65(4, Suppl)A11-A17

33 Doyle JR. Forearm. En: Doyle JR, Botte MJ, editors. Surgical anatomy of the hand and upper extremity. Philadelphia: Lippincott Williams and Wilkins; 2003:407-460

34 Bevin AG. Early tendon transfer for radial nerve transection. Hand 1976;8(02):134-136

35 Ropars M, Dréano T, Siret P, Belot N, Langlais F. Long-term results of tendon transfers in radial and posterior interosseous nerve paralysis. J Hand Surg [Br] 2006;31(05):502-506

36 Altintas AA, Altintas MA, Gazyakan E, Gohla T, Germann G, Sauerbier M. Long-term results and the Disabilities of the Arm, Shoulder, and Hand score analysis after modified Brooks and D'Aubigne tendon transfer for radial nerve palsy. J Hand Surg Am 2009;34(03):474-478

37 Brodman HR. Tendon transfer for old radial nerve paralysis. AMA Arch Surg 1958;76(01):24-27

38 Martínez-Villén G, Muñoz-Marín J, Pérez-Barrero P. Are tendon transfers justified in very old hand radial palsies? J Plast Reconstr Aesthet Surg 2012;65(10):e293-e296 